\title{
COMMUNICATION SYNCHRONIZATION IN CLUSTER-BASED WIRELESS SENSOR NETWORK: A REVIEW
}

\author{
Mahadev Khatke ${ }^{1}$, Pratibha Chavan ${ }^{2}$ \\ ${ }^{1}$ M.E. Student, Department of Information Technology, RMDSSOE, Pune, Maharashtra, India \\ ${ }^{2}$ Asst. Professor, Department of Information Technology, RMDSSOE, Pune, Maharashtra, India
}

\begin{abstract}
A wireless sensor network is acquiring more popularity in different sectors. A scalable, low latency and energy efficient are desire challenges that should meet by wireless sensor network. Clustering permits sensors to systematically communicate among clusters. Cluster based sensor network satisfies these challenges as it provides flexible, energy saving and QoS. The communication efficiency and network performance degrades if the interaction between inter-cluster and intra-cluster communication are not managed properly. The proposed work uses two approaches to solve this problem. At aiming low packet delay and high throughput first approach uses cycle-based synchronous scheduling. By completely removing necessity of communication synchronization second approach send packets with no synchronization delay. The combined scheme can take benefit of both approaches.
\end{abstract}

Keywords: Wireless sensor network, clustering, communication synchronization, QoS. ***

\section{INTRODUCTION}

Wireless sensor networks (WSNs) Provides join between real physical and virtual worlds. WSNs plays an important role in daily life because it having unpredictable applications. It is used to security, transportation, industry science and civil infrastructure. In many cases of WSN replacement or recharge of batteries is impossible so the energy efficiency is major concern.

Some challenges in WSNs :

- $\quad$ Energy Efficiency

- Responsiveness

- Robustness

- Self-Configuration

- Scalability

- Heterogeneity

- Low latency

To meet the challenges like low latency, scalability and energy efficiency clustering is best topology.

Structuring sensor nodes in a one unit and providing organizational structure is done by clusters. Among all topology in wireless sensor network clustering is best topology. With using clustering we are able to achieve high energy efficiency, scalability and prolong network lifetime in WSNs. In cluster structure each cluster has leader, which is called as cluster head $(\mathrm{CH})$ and other nodes are cluster members.

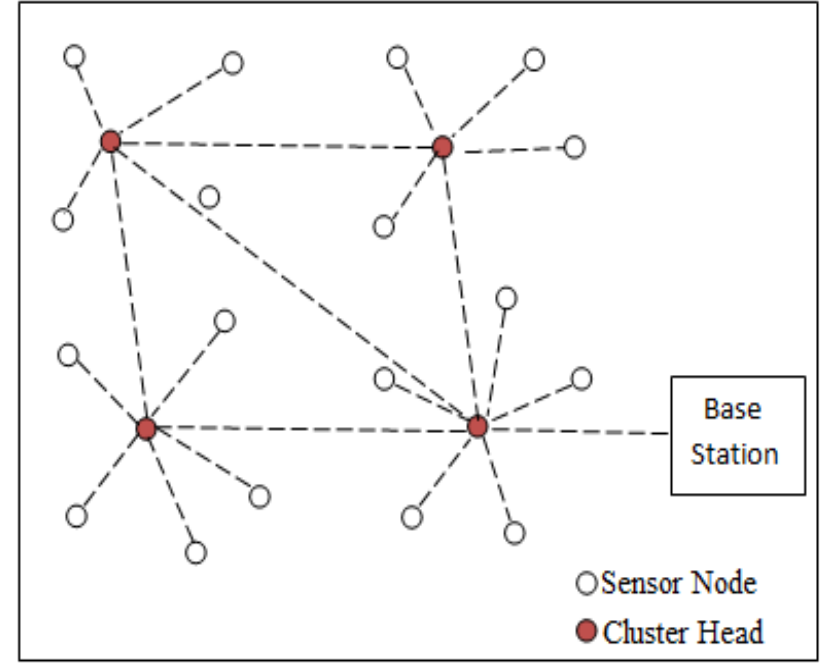

Fig 1 Data communication in clustered network

Sensor nodes collect data periodically and send to corresponding $\mathrm{CHs}$. $\mathrm{CH}$ node aggregate data and transmit them to data sink. Cluster formation process leads to two level hierarchy. $\mathrm{CH}$ at high level and cluster members at low level. Clustering maintain low packet latency. This is possible because data collected by different nodes are combined as aggregated packets at $\mathrm{CHs}$. $\mathrm{CH}$ node act as a gateway between sensor node and base station. Clusterbased WSNs experiences communication synchronization problem because of the complex nature of clustering structure compared to flat topology.

Two types of communication can be possible in WSNs : intra-cluster communication in same cluster and inter-cluster communication in different clusters. The synchronization problem can be managed by grouping all inter-cluster and intra-cluster communications into global and non- 
overlapping periods. This approach definitely avoid the synchronization problem but may cause low channel utilization. To avoid transmission collision, intra-cluster communication uses Time-Division-Multiple-Access (TDMA) based protocols. In a inter-cluster communication TDMA or Carrier-Sense-Multiple-Access(CSMA) based protocols are used. The proposed system used to scheduling schemes to synchronization between inter-cluster and intracluster communication. First approach is TDMA based synchronous scheduling and second is CSMA based new clustering structure (NCS) approach.

\section{RELATED WORK}

Energy efficiency and scalability is achieved by clustering. Cluster formation algorithms can consider two types environment.

- Homogeneous

- Heterogeneous

In homogeneous environment the $\mathrm{CH}$ is ordinary node but in heterogeneous [4] environment the network is deployed in two nodes : basic sensor node and cluster head node. Network functionality is shifted from sensor node to cluster head which reduces the overall cost of network.

There are some cluster formation algorithms as bellow

- Heuristic Schemes: Goal is to search algorithm which have reasonable cluster set up time and finding minimal solution. This type of algorithms are not based on particular metrics

- Weighted Schemes: When sensor loses connectivity with cluster head, this algorithm find new cluster topology and reconfigure network. Uses non periodic procedure to cluster head election. Provide long-lasting architecture when first cluster head process invokes.

- Hierarchical Schemes: LEACH [7] is improved approach of conventional clustering approaches. It randomly selects cluster head to increase energy consumption and life time of wireless sensor network. HEED [6] is multi-hop clustering algorithm. To select cluster head HEED considers communication cost and residual energy.

- Grid Schemes: In this algorithms if node form chain from source to sink, exactly 1 node in this transmission time-frame will be transmitting to the base station.

In inter-cluster communication nodes communicate with nodes of another clusters. Various communication protocols are used in cluster-based wireless sensor networks. Intracluster communication protocols is always TDMA [1] based. Inter-cluster communication takes different approach in different work. TDMA schedules minimum latency and rise in power consumption. This node is activated only when it is scheduled to send or receive packets. The proposed approach solve synchronization problem by using two communication scheduling approaches.

\section{TYPES OF SCHEDULING}

Two types of scheduling is proposed in this paper

- $\quad$ Synchronous scheduling.

- Asynchronous scheduling.

The system is considered as follows

- Considered network is WSN with number of nodes.

- Nodes are randomly distributed in 2-D region.

- $\quad$ Stable topology of relay network.

- Clustering topology is already constructed with help of various clustering algorithms as discussed.

- Nodes can transmit different radio channels .

- Length of sensing packet is same in all over network.

Above assumptions are shared by both synchronous and asynchronous scheduling approach.

\subsection{Synchronous Scheduling}

It uses TDMA based approach called cycle-based scheduling (CBS). In considered WSN topology of relay network is stable while data gathering. In CBS approach each node has given some fixed interval to send and receive packets. Nodes are activated only within that interval otherwise nodes goes in sleep mode. This approach saves energy of network and minimizes synchronization overhead between two nodes. In intra-cluster communication all transmission is from cluster member cluster head. Each adjacent cluster have given different radio channels so the interference from other cluster is avoided. To avoid switching between intra-cluster and inter-cluster communications fixed period is given to all intra-cluster communications. In TDMA scheme whole period is divided in to number of equal slots and data is directly send from node to cluster head.

The relay network is included in inter-cluster communication. At the time of cluster formation $\mathrm{CHs}$ are well ordered in fixed routing tree. Each $\mathrm{CHs}$ have given time interval and within that interval it should send all the packets including packets collected by own cluster and packets from other $\mathrm{CHs}$.

\subsection{Asynchronous Scheduling}

Asynchronous scheduling is called as New Clustering Scheduling (NCS). NCS eliminate the synchronization problem.

NCS has new clustering structure which removes the need of algorithm to schedule inter-cluster communication. It has new clustering structure includes new node, which has highest residual energy called as relay node as shown in Fig 2. The new clustering structure contains $\mathrm{CHs}$, member nodes, relay nodes. The relay node is nothing but the node which takes part in inter-cluster communication. Cluster members collect data and send it to cluster head then cluster head instead of sending data to next hop it sends data to relay node of its own cluster. Relay node add its own data 
and forwards the packets to next hop relay node. This process is repeated until packets reach to sink. Using NCS the switching between inter-cluster communication and intra-cluster communication is possible which reduce data collection delay.

NCS uses TDMA approach for intra-cluster communication and Carrier-Sense-Multiple-Access (CSMA) for intercluster communication.

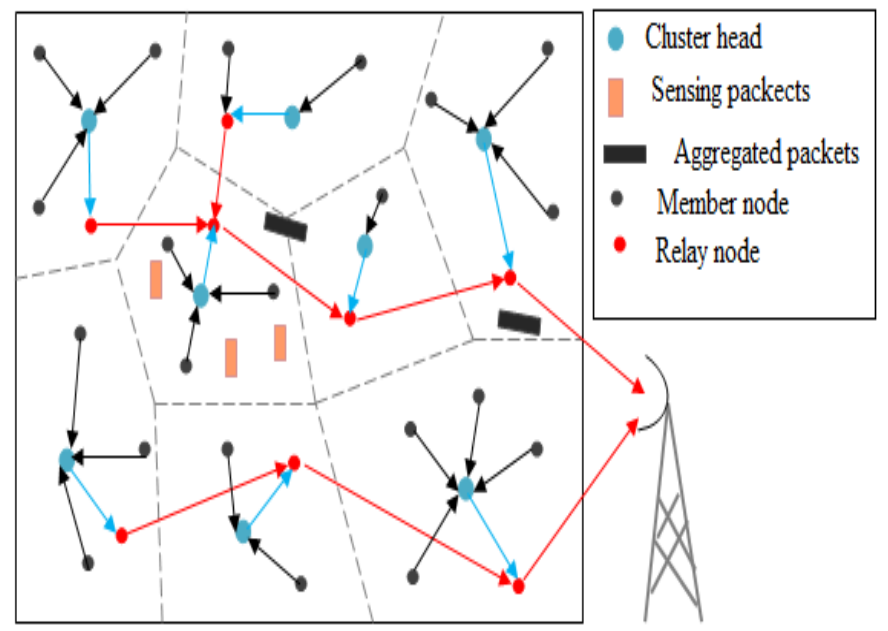

Fig 2 New clustering structure.

CBS uses TDMA which results in energy efficient network. NCS tolerates interference and collision this is possible because it uses CSMA based protocols.

\section{CONCLUSION}

Review includes various cluster formation algorithm. Two types of scheduling approaches are presented: CBS and NCS. In cycle based scheduling fixed interval of time is given to each node and only within than slot of time node is activated to transmit packets. NCS use new node called relay node which remove heavy burden of packet relaying on CHs. Smooth switching between inter-cluster communication and intra-cluster communication is possible. These approaches gives WSNs as fundamental network in cyber physical system.

\section{ACKNOWLEDGEMENTS}

I would like to extend my gratitude to many people who helped me to bring this paper fruition. First I would like to thank my guide Pratibha Chavan. I am so deeply grateful for her help, professionalism, and valuable guidance throughout this paper. I would also like to thank to my friends and college. This accomplishment would not have been possible without them.

\section{REFERENCES}

[1]. L. Shi and A. O. Fapojuwo, "TDMA scheduling with optimized energy efficiency and minimum delay in clustered wireless sensor networks," IEEE Trans. Mobile Computer., vol. 9, no. 7, pp. 927-940, Jul. 2010.
[2]. S. C. Ergen and V. Pravin, "TDMA scheduling algorithms for wireless sensor networks," Wireless Netw., vol. 16, no. 4, pp.985_997, 2010.

[3]. L. Sha, S. Gopalakrishnan, X. Liu, and Q. Wang, "Cyber physical systems: Anewfrontier," in Machine Learning Cyber Trust New York, NY, USA: SpringerVerlag, 2009, pp. 3-13.

[4]. Z. Zhang, M. Ma, and Y. Yang, "Energy efficient multihop polling in clusters of two-layered heterogeneous sensor networks," IEEET rans. Comput., vol. 57, no. 2, pp. 231245, Feb. 2008 .

[5]. M. Lotfinezhad, B. Liang, and E. S. Sousa, "Adaptive cluster-based data collection in sensor networks with direct sink access," IEEE Trans. Mobile Comput., vol. 7, no. 7, pp. 884-897, Jul. 2008

[6]. O. Younis and S. Fahmy, "HEED: A hybrid, energyefficient, distributed clustering approach for ad hoc sensor networks," IEEE Trans. Mobile Comput., vol. 3, no. 4, pp. 366-379, Oct./Dec. 2004.

[7]. W. R. Heinzelman, A. Chandrakasan, and H. Balakrishnan, "Energy efficient communication protocol for wireless microsensor networks," in Proc. 33rd Annu. Hawaii Int. Conf. Syst. Sci., Jan. 2000, pp. 1-10.

[8]. I. Rhee, A.Warrier, M. Aia, J. Min, and M. L. Sichitiu, "Z-MAC: A hybrid MAC for wireless sensor networks," IEEE/ACM Trans. Netw., vol. 16, no. 3, pp. 511_524, Jun. 2008.

[9]. E. Felemban, C. G. Lee, and E. Ekici, “MMSPEED: Multipath multi-SPEED protocol for QoS guarantee of reliability and timeliness in wireless sensor networks," IEEE Trans. Mobile Comput., vol. 5, no. 6, pp. 738_754, Jun. 2006.

[10]. T. He, J. A. Stankovic, C. Lu, and T. Abdelzaher, "SPEED: A stateless protocol for real-time communication in sensor networks," in Proc. ICDCS, May 2003, pp. 46-55.

\section{BIOGRAPHIES}



Mahadev Khatke has obtained Bachelor of Engineering degree from Dr. BAMU, Aurangabad . Now M.E Student at RMD Sinhgad School of Engineering, Pune University, Maharashtra, India

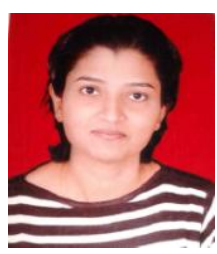

Pratibha Chavan is Assistant Professor at RMD Sinhgad School of Engineering, Pune University, Maharashtra, India. 